ISSN 1808-3765

\title{
COEFICIENTES DE CULTURA PARA A MELANCIEIRA IRRIGADA POR GOTEJAMENTO NO VALE DO GURGUEIA - PI
}

\section{JOSÉ ORLANDO PIAUILINO FERREIRA ${ }^{1}$; LUIZ CARLOS PAVANI ${ }^{2}$ E EDSON ALVES BASTOS ${ }^{3}$}

${ }^{1}$ Colégio Agrícola de Bom Jesus, Universidade Federal do Piauí, Rodovia Municipal Bom Jesus-Viana, 64900000, Bom Jesus - PI, orlando@ufpi.edu.br

${ }^{2}$ Departamento de Engenharia Rural, Faculdade de Ciências Agrárias e Veterinárias, Universidade Estadual Paulista, Via de acesso Prof. Paulo Donato Castellane, s/n, 14884-900, Jaboticabal - SP, lcpavani@fcav.unesp.br ${ }^{3}$ Embrapa Meio-Norte, Av. Duque de Caxias, 5650, CEP: 64006-220, Teresina-PI, edson@cpamn.embrapa.br

\section{RESUMO}

A obtenção de valores locais dos coeficientes de cultura é importante para a estimativa mais acurada das necessidades de irrigação. Com esse objetivo foram determinados os coeficientes de cultura locais - simples $(\mathrm{Kc})$ e basais $(\mathrm{Kcb})$ - da melancieira irrigada por gotejamento no Vale do Gurgueia - PI. A evapotranspiração de referência diária $\left(\mathrm{ET}_{0}\right)$ foi determinada pelo somatório dos valores horários de $\mathrm{ET}_{0}$ aplicando-se o método de Penman-Monteith parametrizado pela FAO com dados climáticos obtidos em estação automática instalada na área. A evapotranspiração diária da cultura foi medida em três lisímetros com células de carga. Visando alta produtividade de frutos nesta região, recomendamos que sejam utilizados para o planejamento e manejo da irrigação os seguintes valores locais de $\mathrm{Kc}$ e de $\mathrm{Kcb}$, respectivamente: fase inicial (estabelecimento da cultura) - 0,34 e 0,24; fase intermediária (crescimento e maturação dos frutos) - 1,16 e 1,10; fase final (colheita) -0,93 e 0,86.Esses valores das fases inicial e intermediária são superiores e os da fase final não diferem dos de Kc e de Kcb já ajustados para as variáveis climáticas locais,altura média da cultura e para a duração da fase final, conforme metodologia da FAO apresentada no Boletim 56 de Irrigação e Drenagem.

Palavras-chave: Citrulluslanatus, evapotranspiração, Kc basal, lisímetro, semiárido.

FERREIRA, J. O. P. F.; PAVANI, L. C.; BASTOS, E. A.

\section{CROP COEFFICIENTS OF DRIP IRRIGATED WATERMELON IN THE GURGUEIA VALE, PIAUI - BRAZIL}

\section{ABSTRACT}

The aim of this work was to determine crop coefficients $(\mathrm{Kc})$ of drip irrigated watermelon in the climatic and growing conditions of the Gurgueia Vale, State of Piaú, Brazil, located at $8^{\circ} 26^{\prime} \mathrm{S}, 43^{\circ} 47^{\prime} \mathrm{W}$ and altitude of $251 \mathrm{~m}$. The reference evapotranspiration $\left(\mathrm{ET}_{0}\right)$ was determined by the sum of hourly values of $\mathrm{ET}_{0}$ obtained by the Penman-Monteith method parameterized by FAO with climatic data obtained from an 
automatic weather station. The daily crop evapotranspiration (ETc) was measured by three weighing lysimeters of load cells. Aiming high fruit yield in this region, we recommend that the following local values of $\mathrm{Kc}$ and $\mathrm{Kcb}$ be used for planning and management of irrigation, respectively: initial stage (crop establishment) - 0.34 and 0.24 ; intermediate stage (growth and fruit maturation) - 1.16 and 1.10; end stage (harvest) - 0.93 and 0.86 . These Kc values of initial and intermediate phases are statistically higher than the values of $\mathrm{Kc}$ and $\mathrm{Kcb}$ already adjusted according to the methodology presented in the FAO Irrigation and Drainage Paper 56. The values of $\mathrm{Kc}$ and $\mathrm{Kcb}$ at the end phase are not statistically different from the FAO values.

Keywords: Citrullus lanatus, semi-arid, evapotranspiration, lysimeters, basal Kc.

\section{INTRODUÇÃO}

Em cultivos manejados com irrigação, adubação e tratamento fitossanitário eficientes e com espaçamento adequado, a produtividade da melancieira pode chegar a cerca de50 $\mathrm{Mg} \mathrm{ha}^{-1}$ (Miranda et al., 2005). No entanto, em 2008 a produtividade média de frutos no Estado do Piaú foi de cerca de $23 \mathrm{Mg} \mathrm{ha}^{-1}$ e em 2010 cresceu para $26 \mathrm{Mg} \mathrm{ha}^{-1}$, enquanto que no Ceará a produtividade em 2010 foi de $41 \mathrm{Mg} \mathrm{ha}^{-1}$. Nos municípios do Vale do Gurgueia, no interior do Piauí, as produtividades variaram de 24 a $30 \mathrm{Mg} \mathrm{ha}^{-1}$ (Instituto Brasileiro de Geografia e Estatística, 2008, 2010).

De acordo com Doorenbos \& Kassan (1994), as necessidades de água para a melancieira, considerando um ciclo de 100 dias, varia de 400 a $600 \mathrm{~mm}$, variaçãoesta de $200 \mathrm{~mm}(+50 \%$ em relação ao menor valor), possivelmente em função de variações de clima, de solo, de manejo cultural e de cultivares. Assim, tendo em vista a necessidade atual e futura de se usar eficientemente a água, devido ao agravamento da escassez desse líquido,estimar o mais precisamente possível o consumo real de água de uma cultura em função das condições locais de solo, clima e cultivo, proporciona menos desperdício, maior racionalidade no planejamento do uso da água como fator de produção e, possivelmente, maior retorno econômico da cultura irrigada.

A quantidade de água a ser fornecida a uma culturapara repor a água evapotranspirada, pode ser estimada através do produto entre a evapotranspiração de referência $\left(\mathrm{ET}_{0}\right)$ e o coeficiente de cultura $(\mathrm{Kc})$, sendo este variável com o estádio de desenvolvimento da cultura, condições de umidade do solo, clima local, frequência de chuva e/ou irrigação e com as práticas de manejo.O teor elevado de sais na água de irrigação e/ ou no solo, pode também dificultar a absorção de água pelas raízes, diminuindo a evapotranspiração da cultura e, consequentemente, modificando os valores de Kc (Figueirêdo et al., 2009). Conceitualmente, o Kc resume em suas diferenças físicas e fisiológicas entre um determinado cultivo e o de referência (Allen et al., 2006a), representando, assim, a utilização de água por uma cultura específica sendo,por isso, o conhecimento dos seus valores,necessário para a estimativa precisa e específica das necessidades de irrigação (Kashyap \& Panda, 2001), principalmente nas fases do ciclo da cultura em que o déficit hídrico no solo pode reduzir significativamente a produtividade da cultura.

Para manejo de irrigação a utilização do Kc simples ou único, que representa os valores médios por fase de desenvolvimento da cultura, dos valores diários de Kc, obtidos em função das condições locais de clima, solo, cultivo e frequência de irrigação e/ou chuva, são suficientes; no entanto, para o conhecimento mais preciso do consumo real diário de água por 
uma cultura, com o fim de estudos pormenorizados de balanço hídrico diário para pesquisa ou para um manejo mais racional da irrigação, o conhecimento dos valores de Kc basal para que se possa compor o Kc dual e estimar a redução no consumo de água pela cultura emcada uma das fases de desenvolvimento, em função das restrições hídricas no solo (Allen et al., 2006a), é fundamental.

O objetivo deste trabalho foi obter os coeficientes de cultura, simples e basal, para a cultura da melancieira irrigada por gotejamento, nas condições do Vale do Gurgueia-PI, como subsídio para manejo de irrigação dessa cultura na região com vista a um cultivo de alta produtividade de frutos.

\section{MATERIAL E MÉTODOS}

O trabalho foi conduzido na área experimental da Embrapa Meio-Norte no município de Alvorada do Gurguéia- PI, com coordenadas geográficas $8^{\circ} 26^{\prime} \mathrm{S}$ de latitude, $43^{\circ} 47^{\prime} \mathrm{Wde}$ longitude e $251 \mathrm{~m}$ de altitude.O clima da região segundo classificação de Köppen é Aw (tropical chuvoso). A precipitação pluvial média anual é de $1.200 \mathrm{~mm}$ e a temperatura média anual é de $26,2^{\circ} \mathrm{C}$ (Lima\& Assunção, 2002), valor esse dentro do intervalo entre $18{ }^{\circ} \mathrm{C}$ e 35 ${ }^{\circ} \mathrm{C}$, respectivamente, temperaturas basais inferior e superior para a melancieira (Onsinejad \& Abak, 1999).

O solo da área experimental é um Latossolo Amarelo (Embrapa Solos, 2006) de textura franco-arenosa na camada de 0 a $0,4 \mathrm{~m}$ de profundidade $\left(56,3 \mathrm{~g} \mathrm{~kg}^{-1}\right.$ de areia grossa, 23, $8 \mathrm{~g} \mathrm{~kg}^{-1}$ de areia fina, 3,3 $\mathrm{g} \mathrm{kg}^{-1}$ de silte e $16,6 \mathrm{~g} \mathrm{~kg}^{-1}$ de argila). Foram utilizados três lisímetros de pesagem, cada um com área interna de $2,25 \mathrm{~m}^{2}(1,50 \mathrm{~m} \mathrm{x} \mathrm{1,50} \mathrm{m)} \mathrm{e}$ profundidade de $1,0 \mathrm{~m}$ e área efetiva para evaporação de $1,70 \mathrm{~m} \times 1,70 \mathrm{~m}\left(2,89 \mathrm{~m}^{2}\right)$, área esta que foi a utilizada para os cálculos (Allenet al., 2011). Cada lisímetro estava conectado a uma célula de carga e a um sistema automático de coleta e armazenamento de dados modelo CR23X (Campbell Scientific) e foram previamente calibrados individualmente.

O preparo do solo da área foi realizado no início do mês de julho de 2008 , de forma semelhante nos lisímetros e na área externa aos lisímetros (bordadura), após o que foram retiradas amostras indeformadas de solo de cada um dos lisímetros e na bordadura, nas profundidades de $0,10 \mathrm{~m}$ e $0,30 \mathrm{~m}$, representando, respectivamente, os perfis de 0 a $0,20 \mathrm{~m}$ e de 0,20 a $0,40 \mathrm{~m}$, para a determinação das umidades na capacidade de campo $(\theta \mathrm{cc})$ e no ponto de murcha permanente $(\theta \mathrm{pmp})$. Foram encontrados valores médios de $\theta \mathrm{cc}$ de $0,1747 \mathrm{~cm}^{3} \mathrm{~cm}^{-3}$ e de $\theta$ pmp de $0,0507 \mathrm{~cm}^{3} \mathrm{~cm}^{-3}$ para o primeiro perfil do solo, e $\theta \mathrm{cc}$ de $0,1690 \mathrm{~cm}^{3} \mathrm{~cm}^{-3}$ e $\theta \mathrm{pmp}$ de $0,0533 \mathrm{~cm}^{3} \mathrm{~cm}^{-3}$ para o segundo perfil.Em sulcos espaçados de 2,0 $\mathrm{m}$, foi realizada, um dia antes da semeadura, adubação com $20 \mathrm{~kg} \mathrm{ha}^{-1}$ de $\mathrm{N}$ (ureia), $80 \mathrm{~kg} \mathrm{ha}^{-1}$ de $\mathrm{P}_{2} \mathrm{O}_{5}$ (superfosfato simples) e $40 \mathrm{~kg} \mathrm{ha}^{-1}$ de $\mathrm{K}_{2} \mathrm{O}$ (cloreto de potássio). Também foram adicionados $10 \mathrm{~kg} \mathrm{ha}^{-1} \mathrm{de}$ FTE BR 12 como fonte de micronutrientes $(9 \% \mathrm{Zn}, 1,8 \% \mathrm{~B}, 0,8 \% \mathrm{Cu}, 2 \% \mathrm{Mn}, 3,5 \% \mathrm{Fe}$ e $0,1 \% \mathrm{Mo})$.

A semeadura da cultivar Crimson Sweet foi realizada no dia 15 de julho de 2008, colocando-setrês sementes por metro de sulco. Dez dias depois de emergidas as plantasfoi realizadodesbaste deixando-se apenas uma planta por metro de sulco, resultando em uma população de 6.336 plantas em área total cultivadade $12.672 \mathrm{~m}$. Aos 25 e 40 DAS procedeuseàs adubações de cobertura com $80 \mathrm{~kg} \mathrm{ha}^{-1}$ de $\mathrm{N}$ (ureia), e $80 \mathrm{~kg} \mathrm{ha}^{-1}$ de $\mathrm{K}_{2} \mathrm{O}$ (cloreto de potássio). Durante a condução do experimento foi efetuado controle fitossanitário preventivo contra pragas e doenças aplicando-se os seguintes defensivos: Carbomax 500 SC (Carbendazim) - 20mL/20L; Actara 250 WG (Thiamethoxam) - $20 \mathrm{~g} / 20 \mathrm{~L}$; Recop 
(Oxicloreto de cobre) - $50 \mathrm{~g} / 20 \mathrm{~L}$; Score (Difenoconazol) - $6 \mathrm{~mL} / 20 \mathrm{~L}$; Decis $25 \mathrm{CE}$ (Deltametrina) - $6 \mathrm{~mL} / 20 \mathrm{~L}$ e Ridomil Gold (Metalxil-M + Ditiocarbamato + Mancozeb) $60 \mathrm{~mL} / 20$ L.A colheita foi realizada no dia 24 de setembro de 2008, aos 72 dias após a semeadura (72 DAS).

A cultura foi irrigada por gotejamento com uma linha porta emissores por linha de plantas, com gotejadores de vazão média de $2,55 \mathrm{~L} \mathrm{~h}^{-1}$ a $200 \mathrm{kPa}$ de pressão,espaçados de $0,5 \mathrm{~m}$ ao longo da linha.

No interior de cada lisímetro, após o desbaste, havia uma planta no centro já que cada planta ocupava uma área de $2,0 \mathrm{~m}^{2}$ e a área interna dos lisímetros na superfície do solo era de $2,25 \mathrm{~m}^{2}$. As ramas dessas plantas foram conduzidas de forma a ficarem uniformemente distribuídas na superfície do solo e as folhas somente no interior dos lisímetros.

$\mathrm{O}$ ciclo da cultura foi dividido em 4 fases, inicialmente divididas em períodos de 20 dias cada, baseada em um ciclo de aproximadamente 80 dias para a cultura na região. A duração de cada fase foi posteriormente corrigida, na medida em que a cultura se desenvolvia, em função do comportamento da curva de ETc obtida com os lisímetros, para a determinação dos valores locais de Kc simples (Kc) e de Kc basal (Kcb)em cada uma dessas fases.

Foi calculado diariamente em cada lisímetro e na bordadura externa o armazenamento real diário de água no solo (RADa) desde 0 até $0,30 \mathrm{~m}$ de profundidade, em camadas de 0,10 $\mathrm{m}$, utilizando-se os valores diários de umidade volumétrica do solo obtidos com sonda de capacitância Diviner $2000^{\circledR}$ (Sentek Environmental Technologies) previamente calibrada para o solo da área (Andrade Júnior et al., 2006). A leitura de umidade do solo era feita nos tubos de acesso para a sonda instalados no interior do bulbo molhado do gotejador próximo à planta, com as quais foi calculada na bordadura e em cada lisímetro, a RADa e esta era comparada com a reserva de água diária crítica (RADc), a fim de verificar se a irrigação aplicada mantinha a RADa com valores iguais ou maiores aos da RADc,até $0,30 \mathrm{~m}$ de profundidade para garantir que a ETc das plantas nos lisímetros e fora deles não era restringida pelo solo.

Para determinar a água total disponível no solo (CAD - Equação 1) e a RADc (Equação 2) e a RADa (Equação 4) como percentuais em relação à CAD, nas fases I, II e III, considerou-se $0,10 \mathrm{~m}, 0,20 \mathrm{~m}$ e $0,30 \mathrm{~m}$, respectivamente, a profundidade efetiva do sistema radicular da cultura para essas fases (Albuquerque, 2007). O fator crítico (p) de esgotamento de água no solo utilizado para a estimativa diária da RADc foi corrigido para valores de ETc diária diferentes de 5,0 $\mathrm{mm} \mathrm{dia}^{-1}$ com a Equação (3), partindo-se do valor de p igual a 0,4 para a uma ETc de 5,0 $\mathrm{mm} \mathrm{dia}^{-1}$, conforme o Boletim 56 de Irrigação e Drenagem da FAO (Allen et al., 2006a).

$$
\begin{aligned}
& \mathrm{CAD}=(\theta \mathrm{cc}-\theta \mathrm{pmp}) \mathrm{Zi} \\
& \mathrm{RADc}=(1-\mathrm{p}) \mathrm{CAD} \\
& \mathrm{p}=0,4+0,04(5,0-\mathrm{ETc}) \\
& \mathrm{RADa}=(\theta \mathrm{a}-\theta \mathrm{pmp}) \mathrm{Zi}
\end{aligned}
$$

Em que: $\theta$ cc é o conteúdo volumétrico de água no solo na capacidade de campo e na camada $\mathrm{Zi}$; $\theta$ pmp é o conteúdo volumétrico de água no solo no ponto de murchamento permanente e na camada $\mathrm{Zi}$; $\mathrm{Zi}$ é a profundidade da camada de solo $(\mathrm{mm})$ na fase i $(\mathrm{i}=\mathrm{I}, \mathrm{Zi}=100 \mathrm{~mm}$; i = II, $\mathrm{Zi}=200 \mathrm{~m} ; \mathrm{i}=\mathrm{III}, \mathrm{Zi}=300 \mathrm{~mm}$ ); $\theta \mathrm{a}$ é a umidade atual do solo medida na camada $\mathrm{Zi}$. 
No centro da área experimental estava instalada uma estação meteorológica automática modelo CR23X(Campbell Scientific), composta por sensores de temperatura ( $\mathrm{T}$, $\left.{ }^{\circ} \mathrm{C}\right)$ e umidade relativa do ar (UR, \%); radiação solar global (Rs, $\mathrm{MJ} \mathrm{m}^{-2} \mathrm{dia}^{-1}$ ); velocidade do vento $\left(\mathrm{U}_{2}, \mathrm{~m} \mathrm{~s}^{-1}\right)$ e precipitação pluvial $(\mathrm{P}, \mathrm{mm})$. Os valores médios dessas variáveis eram armazenados no "datalloger" a cada 15 minutos e os valores médios, a cada hora, os quais foram utilizados para as estimativas horárias da evapotranspiração de referência pelo método de Penman-Montheith de acordo com a equação (5) (Allenet al., 2006b). A estimativa em escala diária da $\mathrm{ET}_{0}$ foi obtida do somatório dos 24 valores horários de $\mathrm{ET}_{0}$.

$$
\mathrm{ET}_{0} \mathrm{PM}_{(\mathrm{h})}=\frac{0,408 \Delta(\mathrm{Rn}-\mathrm{G})+\gamma \frac{\mathrm{Cn}}{\mathrm{T}+273} \mathrm{U}_{2}\left(\mathrm{e}_{\mathrm{s}}-\mathrm{e}_{\mathrm{a}}\right)}{\Delta+\gamma\left(1+\mathrm{Cd} \mathrm{U}_{2}\right)}(5)
$$

Em que: $\mathrm{ET}_{0} \mathrm{PM}_{(\mathrm{h})}$ é a evapotranspiração de referência para período horário, mm; Rn é o saldo de radiação, $\mathrm{MJ} \mathrm{m}^{-2}$; Gé ofluxo horário de calor do solo, $\mathrm{MJ} \mathrm{m}^{-2}$; $\mathrm{T}$ é atemperatura horária média do ar a $2 \mathrm{~m}$ de altura, ${ }^{\circ} \mathrm{C} ; \mathrm{U}_{2}$-velocidade horária média do vento a $2 \mathrm{~m}$ de altura, $\mathrm{m} \mathrm{s}^{-1}$; $\mathrm{e}_{\mathrm{s}}$ é apressão de saturação de vapor à temperatura $\mathrm{T}, \mathrm{kPa}$; $\mathrm{e}_{\mathrm{a}}$ é a pressão atual de vapor, $\mathrm{kPa} ;\left(\mathrm{e}_{\mathrm{s}}\right.$ - $\mathrm{e}_{\mathrm{a}}$ ) é o déficit de pressão de vapor, $\mathrm{kPa} ; \Delta$ é a declividade da curva de pressão de vapor em função da $\mathrm{T}, \mathrm{kPa}{ }^{\circ} \mathrm{C}^{-1} ; \gamma$ é aconstante psicrométrica, $\mathrm{kPa}{ }^{\circ} \mathrm{C}^{-1}$; $\mathrm{Cn}$ é 37 e $\mathrm{Cd}$ é 0,24 nos horários durante o dia em que $\mathrm{Rn}>0$ e 37 e 0,96 , respectivamente, para os horários noturnos (Allen et al., 2006b). Com exceção de $\mathrm{T}$ e $\mathrm{U}_{2}$ que foram medidos, os demais parâmetros da expressão (5) foram estimados por meio dos algoritmos apresentados no Boletim FAO-56 (Allenet al., 2006a).

$$
\mathrm{ET} 0 \mathrm{PM}=\sum_{\mathrm{i}=1}^{24} \mathrm{ET}_{0} \mathrm{PM}_{(\mathrm{h}) \mathrm{i}}(6)
$$

Em que: $\mathrm{ET}_{0} \mathrm{PM}$ é a evapotranspiração diária para a grama como cultura de referência, $\mathrm{mm}$.

Com a razão entre os valores médios diários de ETc dos três lisímetros (ETcLis) e dos valores diários de $\mathrm{ET}_{0} \mathrm{PM}$ foram obtidos os valores diários locais de coeficiente de cultura que, em cada uma das fases definidas, resultaram nos valores médios locais de Kc simples (Kc) e basais $(\mathrm{Kcb})$.A média desses valores em cada fase foi comparadaestatisticamente pelo teste $\mathrm{t}$ de Student, a $1 \%$ e $5 \%$ de probabilidade, com os propostos para a melancieira no Boletim 56 da FAO e ajustadospara condições de umidade relativa média do ar, velocidade média do vento e altura da cultura diferentes das condições padrão (Allen et al., 2006a).

Foi também determinado o índice de cobertura do solopelas plantas (IC, \%),fotografando-se as áreas delimitadas pelos lisímetros e pelas parcelas de bordadura delimitadas com as mesmas dimensões internas dos lisímetros $(1,50 \mathrm{~m} \times 1,50 \mathrm{~m}) \mathrm{e}$, posteriormente, fez-se a relação percentual entre a área coberta pela planta (Apl) e a área dos lisímetros ou das áreas das parcelas nas bordaduras (A). As fotografias foram realizadas duas vezes por semana de uma posição pré-fixada, próximo de cada lisímetro e de cada parcela da bordadura, com uma máquina fotográfica SONY DSC-S600 6.0 megapixels e com aproximação de $3 \mathrm{X}$ (zoom). A Apl foi obtida projetando-se em papel milimetrado a área foliar da planta fotografada e a área do lisímetro ou da parcela da bordadura (A). Desse modo, faziase a contagem das quadrículas referentes à área do lisímetro e/ou bordadura e à área foliar, e a razão entre elas fornecia o IC. 


\section{RESULTADOS E DISCUSSÃO}

O ciclo da cultura foi contabilizado como o período decorrido desde a semeadura(1507-2008) até a colheita dos frutos (24-09-2008; 72 dias após a semeadura - 72 DAS) e foi dividido em quatro fases: I - fase inicial ou de estabelecimento da cultura; II - fase de crescimento vegetativo acelerado, florescimento e de crescimento dos frutos; III - fase intermediária ou de crescimento e maturação dos frutos principais; IV - final da fase III até a colheita. Essas fases foram divididas de acordo com a evolução do consumo de água pela cultura expressa pela curva de evapotranspiração medida (ETcLis - Figura 1).

A irrigação foi aplicada até os 66 DAS.

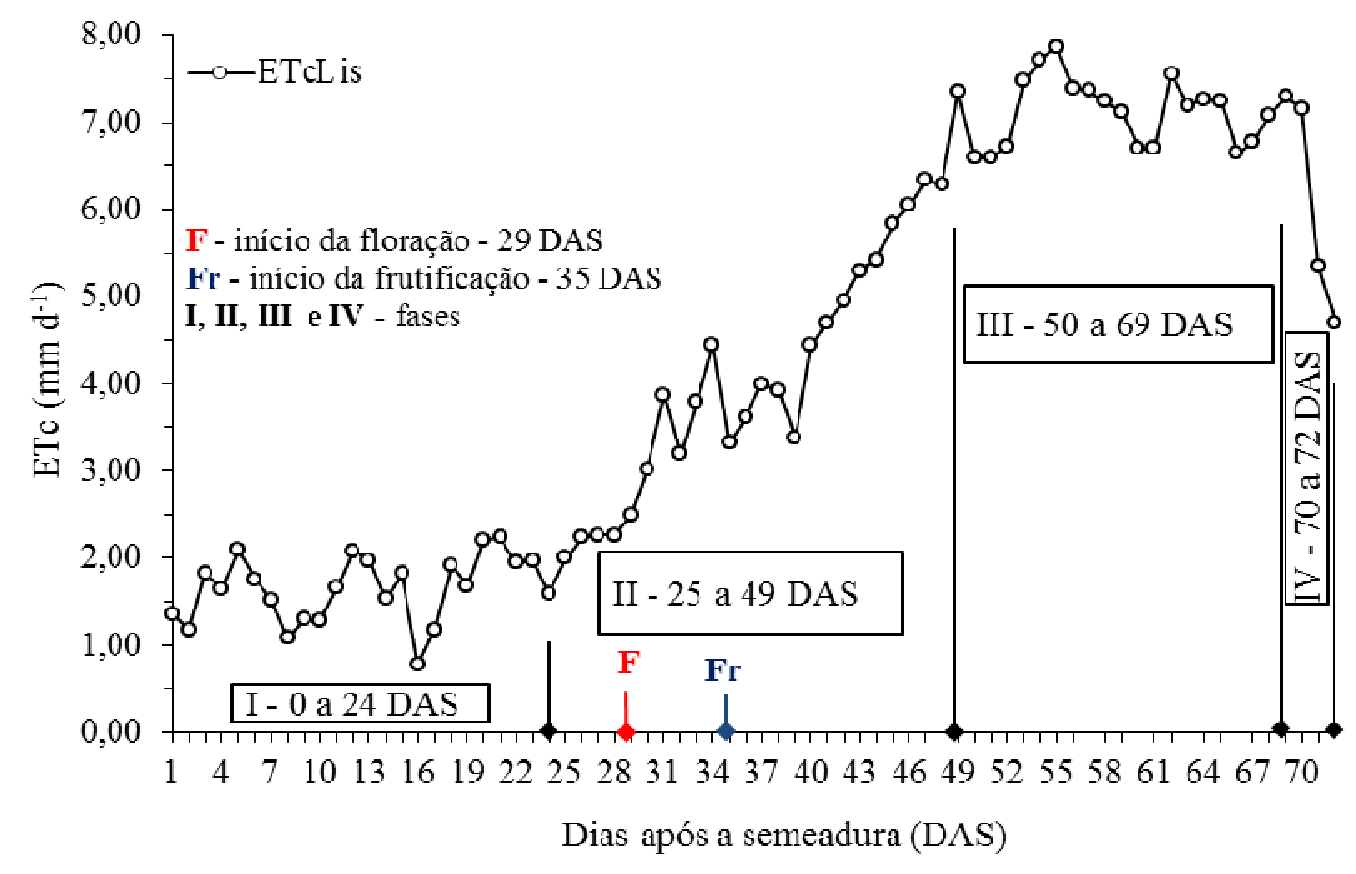

Figura 1. Evapotranspiração diária da cultura de melancia medida com os lisímetros (ETcLis) e divisão do ciclo da cultura em fases de acordo com o comportamento da curva de ETc.

As irrigações diárias mantiveram o armazenamento de água no solo na camada de $0 \mathrm{a}$ 0,30 m de profundidade (RAD real) acima dos níveis críticos de reserva de água disponível no solo (RAD crítica). Na média das fases da cultura o armazenamento de água no solo foi mantido entre $63 \%$ e $81 \%$ da capacidade de água disponível no solo, com fatores reais de consumo ( $\mathrm{p}$ real) inferiores aos críticos ( $\mathrm{p}$ crítico) (Tabela 1). 
Tabela1. Valores médios por fase dos fatores de consumo crítico ( $\mathrm{p}$ crítico), real ( $\mathrm{p}$ real) e das reservas de água disponível como porcentagem da capacidade de água disponível no solo (CAD) crítica (RAD crítica) e real (RAD real) na camada de 0 a $0,30 \mathrm{~m}$ de profundidade em função das irrigações diárias aplicadas até os 66 dias após a semeadura (DAS).

\begin{tabular}{ccccc}
\hline Fases - DAS & $\begin{array}{c}\mathrm{p} \\
\text { crítico }\end{array}$ & $\begin{array}{c}\text { RAD crítica } \\
\text { (\% da CAD) }\end{array}$ & $\begin{array}{c}\mathrm{p} \\
\text { real }\end{array}$ & $\begin{array}{c}\text { RAD real } \\
(\% \text { da CAD })\end{array}$ \\
\hline I - 0 a 24 & 0,52 & 48 & 0,19 & 81 \\
II -25 a 49 & 0,39 & 61 & 0,37 & 63 \\
III -50 a 66 & 0,25 & 75 & 0,19 & 81 \\
\hline Média & 0,38 & 61 & 0,25 & 75 \\
\hline
\end{tabular}

No final da fase I, aos 24 DAS, o índice de cobertura do solo pela cultura (IC) era de 2\% (Figura 2). Doorembos \& Pruitt (1977) e Allen et al. (2006a) adotam IC de 10\% como indicativo do final da fase I. No entanto, quando ocorreu $10 \%$ de IC na área, entre 31 e 35 DAS, a curva de evolução da ETc (Figura 1) já se encontrava em clara ascensão, ou seja, já na fase II.

O final da fase II foi considerado aos 49 DAS pela curva de ETc, com IC de, aproximadamente, $73 \%$. O valor de IC preconizado por Allenet al. (2006a), para o final dessa fase é $80 \%$. Durante esta fase, que durou 25 dias, foram feitas duas adubações de cobertura o que favoreceu o aumento da taxa de crescimento do IC (Figura 2), devido ao aumento da área foliar pelo crescimento e multiplicação das ramas. Em consequência, o consumo diário de água pela cultura foi continuamente ascendente até os 49 DAS (Figura 1).

A fase III teve duração de 20 dias, dos 50 aos 69 DAS, com IC variando de 73 a 96\%. Estes valores altos de IC indicam que a ETc da cultura se mantém elevada, mas depende, principalmente, das flutuações das variáveis climatológicas, pois a expansão da superfície foliar sobre a área de solo, que foi o fator preponderante na fase II para o aumento sucessivo da ETc, já havia atingido o máximo possível. Nessa fase, os frutos que começaram a se desenvolver ainda na fase anterior, atingiram o máximo crescimento e a maturação. Alguns frutos que começaram a crescer a partir de flores que abriram a partir da metade da fase II, na fase III não alcançaram o tamanho e a maturação para serem colhidos no final da fase IV. 


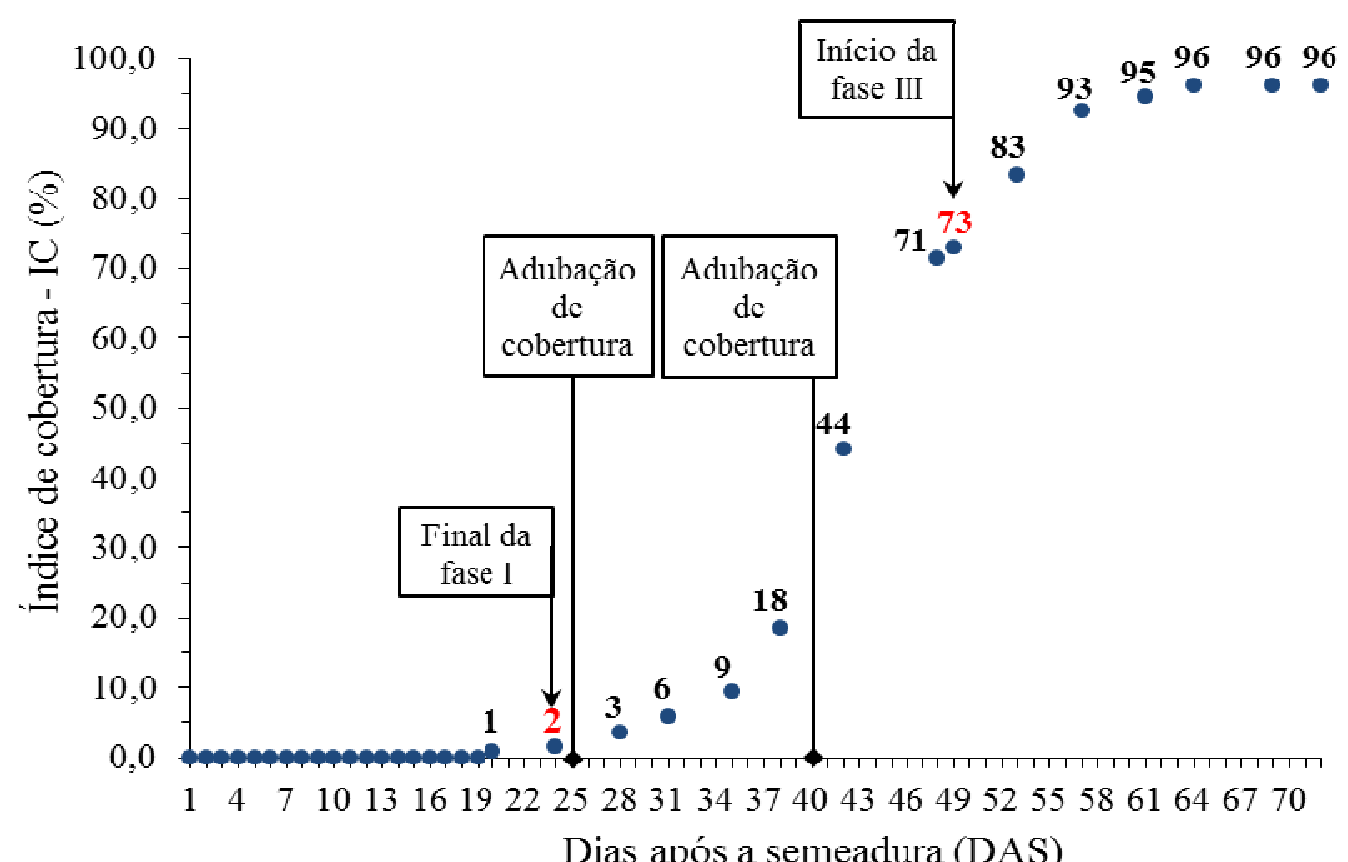

Figura 2. Evolução do índice de cobertura do solo pela vegetação (IC), valores médios entre os IC da área interna e externa aos lisímetros, durante o ciclo da cultura da melancieira e detalhes dos valores do IC no final da fase I e início da fase III e dos dias de adubação de cobertura.

A fase IV teve apenas três dias de duração, dos 70 aos 72 DAS, caracterizando-se por rápida senescência das folhas e, como consequência, queda acentuada da ETc (Figura 1). Aos 72 DAS foram colhidos os frutos que se apresentavam maduros, sobrando nas plantas apenas frutos sem valor comercial. Essa colheita foi a principal, considerando-se encerrado o ciclo de cultivo agronômico da cultura.

As condições meteorológicas médias do ciclo foram favoráveis ao desenvolvimento da cultura, com temperatura do ar (T) acima de $25{ }^{\circ} \mathrm{C}$, umidade relativa do ar (UR) abaixo de $45 \%$, ausência de chuva $(\mathrm{P})$, velocidade média do vento $\left(\mathrm{U}_{2}\right)$ menor do que $2,0 \mathrm{~m} \mathrm{~s}^{-1}$, radiação solar global (Rs) e saldo de radiação (Rn) acima de 20 e de $10 \mathrm{MJ} \mathrm{m}^{-2} \mathrm{~d}^{-1}$, respectivamente. A interação dessas variáveis com as de solo e planta, resultaram em valor médio de evapotranspiração de referência $\left(\mathrm{ET}_{0} \mathrm{PM}\right)$ de $5,51 \mathrm{~mm} \mathrm{~d}^{-1}$, considerado alto (faixa entre 5 e 7 $\mathrm{mm} \mathrm{d}^{-1}$ ) de acordo com o Quadro 9 do Boletim 56 da FAO (Allen et al., 2006a) e em total de $397,05 \mathrm{~mm}$. Com relação à ETc da melancieira os valores total e médio foram 303,68 $\mathrm{mm}$ e $4,22 \mathrm{~mm} \mathrm{~d}^{-1}$, respectivamente, durante os 72 dias de cultivo (Tabela 2). 
Tabela2. Valores das principais variáveis meteorológicas e das evapotranspirações de referência e da cultura, por fase do ciclo e total.

\begin{tabular}{|c|c|c|c|c|c|c|c|c|c|}
\hline \multirow{3}{*}{$\begin{array}{c}\text { Fases do } \\
\text { ciclo }\end{array}$} & \multirow{3}{*}{$\begin{array}{c}\text { Duração } \\
\text { (dias) }\end{array}$} & \multirow{2}{*}{$\begin{array}{c}\mathrm{T} \\
\left({ }^{\circ} \mathrm{C}\right) \\
\end{array}$} & \multirow{2}{*}{$\begin{array}{l}\text { UR } \\
(\%)\end{array}$} & \multirow{2}{*}{$\begin{array}{c}\mathrm{U}_{2} \\
\left(\mathrm{~m} \mathrm{~s}^{-1}\right)\end{array}$} & \multirow{2}{*}{$\begin{array}{c}\mathrm{P} \\
(\mathrm{mm})\end{array}$} & Rs & $\mathrm{Rn}$ & $\mathrm{ET}_{0} \mathrm{PM}$ & ETcLis \\
\hline & & & & & & \multicolumn{2}{|c|}{$\left(\mathrm{MJ} \mathrm{m}^{-2} \mathrm{~d}^{-1}\right)$} & \multicolumn{2}{|c|}{$\left(\mathrm{mm} \mathrm{d}^{-1}\right)$} \\
\hline & & & Média & & Total & & & Média & \\
\hline I & 24 & 25,2 & 48,7 & 1,2 & 0,0 & 20,2 & 10,1 & 4,93 & 1,65 \\
\hline II & 25 & 27,0 & 40,9 & 1,3 & 0,0 & 21,7 & 10,8 & 5,54 & 4,18 \\
\hline III & 20 & 28,5 & 35,4 & 1,4 & 0,0 & 23,4 & 11,7 & 6,17 & 7,13 \\
\hline IV & 3 & 29,8 & 40,9 & 0,7 & 0,0 & 26,3 & 13,2 & 5,65 & 5,74 \\
\hline Média & - & 26,9 & 42,0 & 1,3 & - & 21,9 & 10,9 & 5,51 & 4,22 \\
\hline Total & 72 & - & - & - & 0,0 & 1573,5 & 784,8 & 397,05 & 303,68 \\
\hline
\end{tabular}

$\mathrm{T}$ - temperatura do ar; UR - umidade relativa do ar; $\mathrm{U}_{2}$ - velocidade do vento a $2 \mathrm{~m}$ de altura; $\mathrm{P}$ - chuva; Rs - radiação solar global; $\mathrm{Rn}$ - saldo de radiação; $\mathrm{ET}_{0} \mathrm{PM}$ - evapotranspiração de referência pelo método de Penman-Monteith; ETcLis - evapotranspiração da cultura medida nos lisímetros.

As condições ambientais favoráveis, adequado manejo cultural e o uso da irrigação por gotejamento, contribuíram para a alta produtividade de frutos obtida nesse experimento que foi de 56,95 $\mathrm{Mgha}^{-1}$.

A época de cultivo, localização da área, variedade ou híbrido utilizado, práticas de manejo cultural, as condições hídricas do solo e climatológicas da época, além da metodologia utilizada para a determinação da ETc, podem resultar em valores diferentes para o consumo total de água pela cultura. De acordo com Doorembos \& Kassan (1994), considerando um ciclo total de 100 dias, as necessidades hídricas da melancieira variam de 400 a $600 \mathrm{~mm}$, que estimados para os 72 dias de ciclo total da cultura neste experimento, resultaria em consumo entre 288 e $432 \mathrm{~mm}$. Nesta faixa se encaixa os 303,68 mm de ETc total obtidos neste experimento. No entanto, em região litorânea do Ceará $\left(3^{\circ} 43^{\prime} \mathrm{S}, 38^{\circ} 32^{\prime} \mathrm{W}\right.$ e $19,5 \mathrm{~m}$ de altitude) que é cerca de $5^{\circ}$ mais ao norte, $5^{\circ}$ mais a leste e $231 \mathrm{~m}$ mais baixo do que a área deste experimento em Alvorada do Gurgueia - PI, com a mesma variedade Crimson Sweet, cultivada de outubro a dezembro de 1997, Bezerra\& Oliveira (1999), aplicando a metodologia do balanço hídrico no solo, determinaram que a ETc da cultura durante 59 dias de avaliação foi de $319,2 \mathrm{~mm}$. A cultura foi também irrigada por gotejamento, mas com manejo de irrigação feito com tensiômetros e a semeadura feita em covas espaçadas de $3 \mathrm{~m} \mathrm{x}$ $2 \mathrm{~m}$ e duas plantas por cova (neste experimento foram linhas espaçadas de $2 \mathrm{~m}$ e $1 \mathrm{~m}$ entre plantas na linha, com uma planta por metro). O ciclo total da cultura foi de 65 dias.

$\mathrm{Na}$ mesma área no Ceará, mas com uma variedade de melancia sem sementes, plantio realizado a partir de mudas obtidas em casa de vegetação, no período de 14 de outubro a 13 de dezembro de 2003, e com práticas de adubação, de manejo cultural e de irrigação (esta não especificada pelos autores) distintas das empregadas neste experimento, o ciclo total da cultura foi de 61 dias, e a ETc total desde o transplantio das mudas, obtida por balanço hídrico em lisímetros de drenagem, foi de $277 \mathrm{~mm}$ e a produtividade obtida foi de $27,7 \mathrm{Mg} \mathrm{ha}{ }^{-1}$ (Carvalho et al., 2007).

Também no Ceará ( $3^{\circ} 29^{\prime} \mathrm{S}, 39^{\circ} 09^{\prime} \mathrm{W}$ e altitude de $30 \mathrm{~m}$ ) e com a variedade Crimson Sweet cultivada no espaçamento de $2,0 \mathrm{~m} \times 0,75 \mathrm{~m}$, com duas plantas por cova e irrigada por gotejamento, a ETc total obtida, medida em lisímetros de pesagem, foi de $267 \mathrm{~mm}$ para um ciclo total de 70 dias e produtividade de $57,7 \mathrm{Mg} \mathrm{ha}^{-1}$ (Miranda et al., 2004).

Os valores médios locais de Kc simples e de Kcb foram maiores $(\mathrm{P}<0,01)$ do que os de Kc FAO-56 e Kcb FAO-56 ajustados nas fases I $(n=72)$ e III $(n=60)$. Na fase IV não houve 
diferença estatística $(\mathrm{P}>0,05)$ provavelmente em vista do pequeno número de dados disponíveis nessa fase que foi curta ( $\mathrm{n}=6$ para $\mathrm{Kc}$ e $\mathrm{n}=3$ para $\mathrm{Kcb}$ ) (Tabela 3), diminuindo os graus de liberdade (GL) na análise estatística ( $\mathrm{GL}=5$ para $\mathrm{Kc}$ e 2 para $\mathrm{Kcb}$ ) resultando, por isso,que os valores calculados de t para essas condições eram inferiores aos valores críticos (tc) tabelados para 5\% e $1 \%$ de probabilidade.

Os valores de $\mathrm{Kc}$ e $\mathrm{Kcb}$ médios locais não foram inferiores aos valores máximos limites de Kc em cada fase (Kc máximo; Tabela 4) estimados em função das condições ambientais médias e da altura da cultura (UR mínima, $\mathrm{U}_{2}$ e h) conforme a equação 72 do Boletim FAO-56, o que é compatível com a disponibilidade líquida máxima de energia disponível no meio (Allen et al., 2006a, 2011).

Tabela 3. Coeficientes de cultura médios para as fases I, III e IV do ciclo da melancieira: Kc simples - Boletim FAO-56; tabelado - (KcFAO-56 simples); e ajustado (KcFAO56 simples ajustado); obtidos - (Kc local simples); basais - Boletim FAO-56; tabelado - (KcbFAO-56); e ajustado (KcbFAO-56 ajustado); obtidos - (Kcb local)

\begin{tabular}{ccccccc}
\hline Fases do ciclo & $\begin{array}{c}\text { KcFAO-56 } \\
\text { simples }\end{array}$ & $\begin{array}{c}\text { KcFAO-56 } \\
\text { simples ajustado }\end{array}$ & $\begin{array}{c}\text { Kc local } \\
\text { simples }\end{array}$ & $\begin{array}{c}\text { Kcb } \\
\text { FAO-56 }\end{array}$ & $\begin{array}{c}\text { KcbFAO-56 } \\
\text { ajustado }\end{array}$ & $\begin{array}{c}\text { Kcb } \\
\text { local }\end{array}$ \\
\hline I & 0,40 & $\left(^{*} 0,29 \mathrm{~B}\right.$ & $0,34 \mathrm{~A}$ & 0,15 & $(*) 0,15 \mathrm{~b}$ & $0,24 \mathrm{a}$ \\
II & - & - & - & - & - & - \\
III & 1,00 & $1,10 \mathrm{~B}$ & $1,16 \mathrm{~A}$ & 0,95 & $1,00 \mathrm{~b}$ & $1,10 \mathrm{a}$ \\
IV & 0,98 & $1,07 \mathrm{~A}$ & $0,93 \mathrm{~A}$ & 0,92 & $0,97 \mathrm{a}$ & $0,86 \mathrm{a}$ \\
\hline
\end{tabular}

*)Letras maiúsculas na linha comparam, em cada fase, médias de KcFAO-56 simples ajustado com Kc local simples, e letras minúsculas na linha comparam, em cada fase, médias de KcbFAO-56 ajustado com as de Kcb local. Médias seguidas de letras iguais, maiúsculas ou minúsculas, não diferem entre si pelo teste t de Student $(\mathrm{P}>0,05)$.

Tabela 4. Limite superior do Kc (Kc máximo) em cada fase dos ciclo em função das condições médias de umidade relativa mínima (UR mínima), velocidade do vento a $2 \mathrm{~m}$ de altura $\left(\mathrm{U}_{2}\right)$ e altura da cultura $(\mathrm{h})$, de acordo com a equação 72 do Boletim 56 da FAO.

\begin{tabular}{|c|c|c|c|c|}
\hline \multirow[t]{2}{*}{ Fases do ciclo } & $\begin{array}{c}\text { UR mínima } \\
(\%)\end{array}$ & $\begin{array}{c}\mathrm{U}_{2} \\
\left(\mathrm{~m} \mathrm{~s}^{-1}\right)\end{array}$ & $\begin{array}{l}\mathrm{h} \\
(\mathrm{m})\end{array}$ & \multirow[t]{2}{*}{ Kc máximo } \\
\hline & & Média & & \\
\hline I & 23,3 & 1,2 & 0,15 & 1,22 \\
\hline II & 20,2 & 1,3 & 0,20 & 1,23 \\
\hline III & 15,9 & 1,4 & 0,40 & 1,25 \\
\hline IV & 26,2 & 0,7 & 0,40 & 1,21 \\
\hline
\end{tabular}

As curvas típicas de Kc local simples e de Kcb local para a melancieira foram plotadas juntamente com os valores locais diários de $\mathrm{Kc}$ (Kc local diário) e estão apresentadas na Figura 3. Esta figura poderá servir como base para a estimativa do consumo de água da melancieira cultivar Crimson Sweet e para planejamento de manejo e programação da irrigação dessa cultivar no Vale do Gurgueia - PI. 


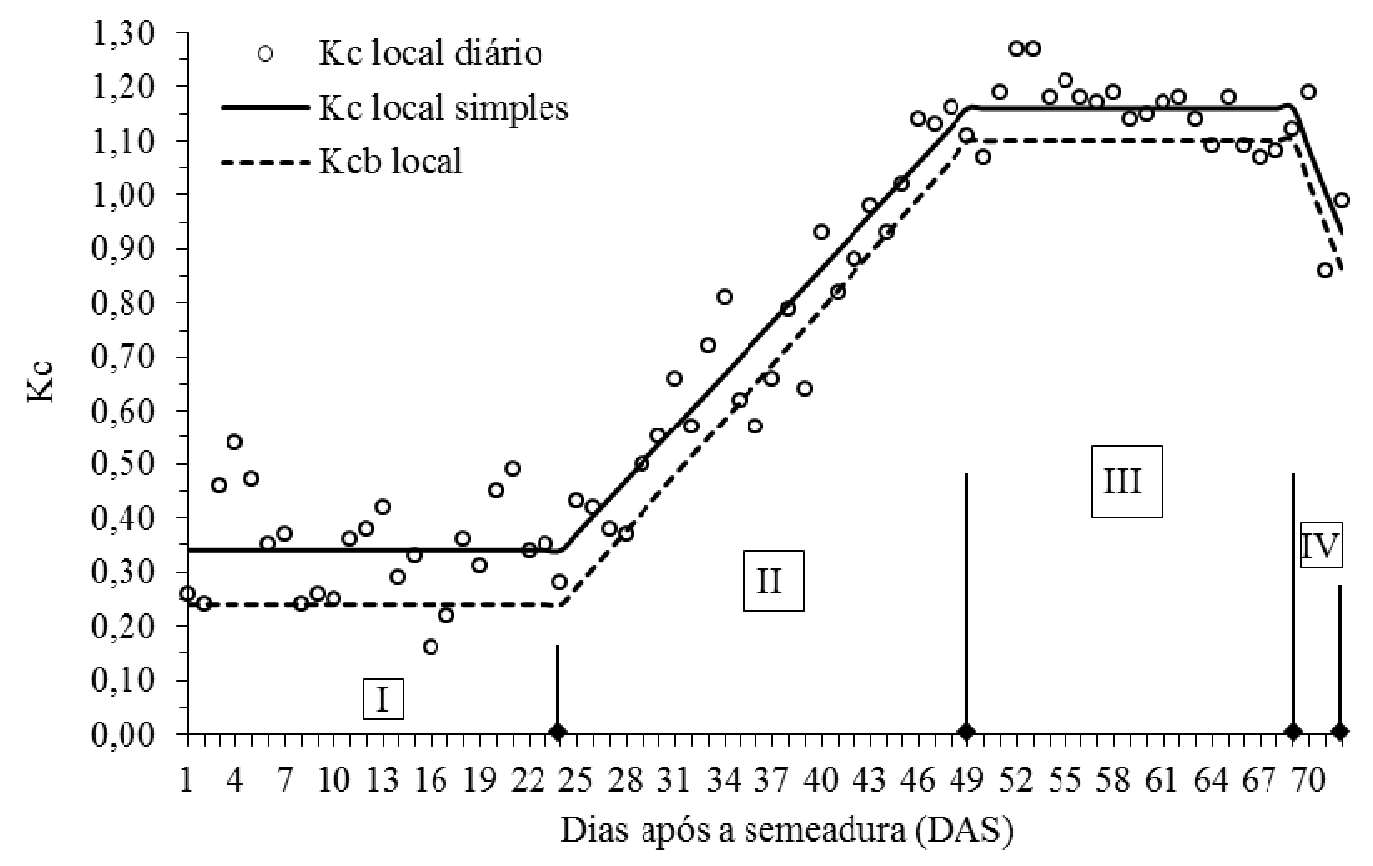

Figura3. Variação diária do coeficiente de cultura (Kc local diário) e curvas de Kc simples (Kc local simples) e de Kc basal (Kcb local) em função das fases da cultura da melancieira.

Os valores de Kc simples obtidos nesta pesquisa foram mais próximos aos obtidos por Bezerra\& Oliveira (1999) em Fortaleza - CE, para a mesma cultivar Crimson Sweet. Os valores obtidos por esses autores foram 0,32 (fase I), 1,27 (fase III) e 0,95 (final da fase IV). Já Mirandaet al. (2004), em Paraipaba - CE, com a mesma cultivar e também irrigada por gotejamento, obtiveram valores de Kc simples semelhantes aos desta pesquisa apenas nas fases I $(0,30)$ e III $(1,15)$; no final da fase IV o valor foi menor $(0,58)$ e praticamente igual $(0,59)$ ao obtido em lisímetros de drenagem por Carvalho et al. (2007) em Fortaleza $\mathrm{CE}$,nessa mesma fase, para a cultivar Honey Ball sem sementes. Nas fases I e III os valores de Kc simples obtidos por esses pesquisadores foram 0,38 e 0,98 .

Em Mossoró - RN, com a cultivar Mickylee irrigada por gotejamento, os valores de Kc resultantes do trabalho de Figueirêdo et al. (2009), para as fases I, III e final da fase IV, foram 0,$23 ; 1,12$ e 0,69 , respectivamente, ou seja, apenas o Kc simples da fase III foi mais próximo ao obtido neste trabalho. Isso ocorre, provavelmente, por que na fase III, independentemente da cultivar, as plantas atingem o valor máximo de índice de área foliar, fazendo com que as características aerodinâmicas do dossel se tornem bastante semelhantes. As condições e tamanho da bordadura da cultura em torno dos lisímetros, a condução da cultura dentro e fora dos lisímetros e o tipo de lisímetro podem determinar valores discrepantes entre as medidas de Kc de uma mesma cultura em locais e épocas diferentes (Allen et al., 2011).

\section{CONCLUSÕES}

Recomendamos que o planejamento e o manejo de irrigação da melancieira cultivar Crimson Sweet no Vale do Gurgueia - PI, irrigada por gotejamento e visando alta 
produtividade de frutos, sejamelaborados considerando os valores de $\mathrm{Kc}$ simples e basal obtidos, que foram: fase I - Kc simples: 0,34; Kc basal: 0,24; fase III - Kc simples: 1,16; Kc basal 1,10; final da fase IV - Kc simples: 0,93; Kc basal: 0,86.

Os valores de Kc simples e de Kc basal das fases I e III foram significativamente maiores do que os valores propostos pela FAO para a melancieira, no Boletim 56, embora não tenham excedido os valores máximos de Kc simples e basal determinados pela disponibilidade de energia no meio . Os valores desses coeficientes para o final da fase IV não diferiram dos propostos no Boletim 56 da FAO.

\section{REFERÊNCIAS}

ALBUQUERQUE, P. E. P. Planilha eletrônica para programação da irrigação em sistemas de aspersão convencional, pivô central e sulcos. Sete Lagoas: Embrapa Milho e Sorgo. 2007. 17 p. (Circular Técnica, 97).

ALLEN, R. G.; PEREIRA, L. S.; HOWELL, T. A.; JENSEN, M. E. Evapotranspiration information reporting: I. Factors governing measurement accuracy. Agricultural Water Management, Amsterdam, v.98, n.6, p.899-920, 2011.

ALLEN, R. G.; PEREIRA, L. S.; RAES, D.; SMITH, M. Evapotranspiración del cultivo: guia para la determinación de los requerimientos de agua de los cultivos.Roma: FAO, 2006a. 298 p. (Estudio Riego y drenaje, 56).

ALLEN, R. G.; PRUITT, W. O.; WRIGHT, J. L.; HOWELL, T. A.; VENTURA, F.; SNYDER, R., ITENFISU, D.; STEDUTO, P.; BERENGENA, J.; YRISARRY, J. B.; SMITH, M.; PEREIRA, L. S.; RAES, D.; PERRIER, A.; ALVES, I.; WALTER, I.; ELLIOT, R. A recommendation on standardized surface resistance for hourly calculation ofreference $\mathrm{ET}_{0}$ by the FAO56 Penman-Monteith method. Agricultural Water Management, Amsterdam, v.81. n.1-2,p.1-22, $2006 b$.

ANDRADE JÚNIOR, A. S.; SILVA, C. R.; DANIEL, R.; CAVALCANTE, R. F.; FIGUEREDO JÚNIOR, L. G. M.; SOUZA, C. F. Calibração de sensor de capacitância para determinação do conteúdo de água em um Latossolo Amarelo. In: CONGRESSO BRASILEIRO DE ENGENHARIA AGRÍCOLA, 35., 2006, João Pessoa. Anais... João Pessoa: SBEA, 2006. 1 CD-ROM.

BEZERRA, F. M. L.; OLIVEIRA, C. H. C. Evapotranspiração máxima e coeficiente de cultura nos estádios fenológicos da melancia irrigada. Revista Brasileira de Engenharia Agrícola e Ambiental, Campina Grande, v.3, n.2, p.173-177, 1999.

CARVALHO, L. C. C.; BEZERRA, F. M. L.; CARVALHO, M. A. R. Evapotranspiração e coeficientes de cultivo da melancieira sem sementes. Revista Ciência Agronômica, Fortaleza, v.39, n.1, p.53-59, 2007.

DOORENBOS, J., KASSAN, A. H. Efeito da água no rendimento das culturas. Estudos FAO - irrigação e Drenagem, no 33, p. 306, 1994. (Traduzido por Gheyi H. R. et al - UFPB). 
DOORENBOS, J., PRUTTI, W. O. Crop water requirements. Rome: FAO, 1977. 179 p. (FAO - Irrigation and Drainage Paper, 24).

EMBRAPA SOLOS. Mapa exploratório-Reconhecimento de solos do município de Alvorada do Gurgueia-PI. Boa Viagem: Embrapa Solos, 2006. Disponível em: $<$ http://www.cnps.embrapa.br/solos>. Acesso em: 20 abr. 2009.

FIGUEIRÊDO, V. B.; MEDEIROS, J. F.; ZOCOLER, J. L.; ESPINOLA SOBRINHO, J. Evapotranspiração da cultura da melancia irrigada com água de diferentes salinidades. Engenharia Agrícola, Jaboticabal, v.29, n.2, p.231-240, 2009.

INSTITUTO BRASILEIRO DE GEOGRAFIA E ESTATÍSTICA. Sistema IBGE de recuperação automática. Rio de Janeiro, 2008. Disponível em:

$<$ http://www.sidra.ibge.gov.br/bda/tabela>. Acesso em: 14 jan. 2010.

INSTITUTO BRASILEIRO DE GEOGRAFIA E ESTATÍSTICA. Produção agrícola municipal: culturas temporárias e permanentes. Brasília, 2010. v.37.89p. Disponível em: $<$ http://www.ibge.gov.br/home/estatistica/economia/pam/2010/PAM2010_Publicacao_compl eta.pdf>. Acesso em: 30 jul. 2012.

KASHYAP, P. S.; PANDA, R. K. Evaluation of evapotranspiration estimation methods and development of crop-coefficients for potato crop in a sub-humid region. Agricultural Water Management, Amsterdam, v.50, n.1, p. 9-25, 2001.

LIMA, M. G., ASSUNÇÃO, H. F. Estimativa da temperatura do ar no Piauí. Teresina: UFPI, 2002. 48 p.

MIRANDA, F. R.; MONTENEGRO, A. A. T.; J. G. OLIVEIRA, J. J. G. Produtividade da melancia irrigada por gotejamento em diferentesespaçamentos de plantio. Revista Ciência Agronômica, Fortaleza, v.36, n.2, p.158-162, 2005.

MIRANDA, F. R.; OLIVEIRA, J. J. G.; SOUZA, F. Evapotranspiração máxima e coeficiente de cultivo para a cultura da melancia irrigada por gotejamento. Revista Ciência Agronômica, Fortaleza, v.35, n.1, p.36-43, 2004.

ONSINEJAD, R.; ABAK, K. Determination of a suitable formula for the calculation of sum growing degree days in watermelon (Citrulluslanatus (thunb,) Mansf,). Acta Horticulturae, Leuven, v.492, p.297-302, 1999. 